\title{
Daratumumab/Dexamethasone/Pomalidomide Regimen
}

National Cancer Institute

\section{Source}

National Cancer Institute. Daratumumab/Dexamethasone/Pomalidomide Regimen. NCI

Thesaurus. Code C160557.

A chemoimmunotherapy regimen consisting of daratumumab, dexamethasone and pomalidomide that can be used in the treatment of relapsed/refractory plasma cell (multiple) myeloma. 